\title{
Intraoperative pathological investigation of recurrent nerve nodal metastasis can guide the decision whether to perform cervical lymph node dissection in thoracic esophageal cancer
}

\author{
YUJI UEDA ${ }^{1}$, ATSUSHI SHIOZAKI ${ }^{1}$, HIROSUMI ITOI ${ }^{2}$, KAZUMA OKAMOTO $^{1}$, HITOSHI FUJIWARA ${ }^{1}$, \\ DAISUKE ICHIKAWA ${ }^{1}$, SHOJIRO KIKUCHI ${ }^{1}$, NOBUAKI FUJI ${ }^{1}$, TSUYOSHI ITOH ${ }^{1}$, \\ TOSHIYA OCHIAI ${ }^{1}$, SHUHEI KOMATSU ${ }^{1}$ and HISAKAZU YAMAGISHI ${ }^{1}$ \\ ${ }^{1}$ Department of Surgery, Division of Digestive Surgery, Kyoto Prefectural University of Medicine Graduate School of \\ Medical Science, 465 Kajii-cho, Kawaramachi-Hirokoji, Kamigyo-ku, Kyoto 602-8566; ${ }^{2}$ Department of Surgery, \\ Meiji University of Oriental Medicine Graduate School of Acupuncture and Moxibustion, \\ Hiyoshi-cho, Nantan, Kyoto 602-0392, Japan
}

Received July 3, 2006; Accepted August 7, 2006

\begin{abstract}
Three-field lymph node dissection has been widely used to treat thoracic esophageal cancer, but is very invasive and can cause serious complications. Whether cervical lymph node dissection should be performed in all patients with thoracic esophageal cancer remains controversial. We pathologically examined the recurrent nerve lymph nodes during surgery in patients with thoracic esophageal cancer to determine the presence or absence of lymph node involvement. In patients without recurrent nerve nodal involvement, cervical lymph node dissection was not performed. Treatment outcomes were analyzed to evaluate whether intraoperative pathological investigation was a useful procedure. Among 71 patients with thoracic esophageal cancer who underwent 3-field lymph node dissection, the rate of cervical lymph node metastasis was $40.9 \%$ in patients with recurrent nerve nodal metastasis on intraoperative pathological investigation, as compared with $10.2 \%$ in patients without recurrent nerve nodal metastasis $(\mathrm{p}=0.007)$. Multiple logistic-regression analysis showed that recurrent nerve nodal metastasis was a strong predictor of cervical lymph node metastasis (odds ratio, 2.98; $95 \%$ confidence interval, 1.139-7.775; $\mathrm{p}=0.03$ ). Among 41 patients who underwent intraoperative pathological investigation, 10 had recurrent nerve nodal metastasis and underwent cervical lymph node dissection. Two of these patients had histological
\end{abstract}

Correspondence to: Dr Yuji Ueda, Department of Surgery, Division of Digestive Surgery, Kyoto Prefectural University of Medicine Graduate School of Medical Science, 465 Kajii-cho, KawaramachiHirokoji, Kamigyo-ku, Kyoto 602-8566, Japan

E-mail: yueda@koto.kpu-m.ac.jp

Key words: esophageal cancer, lymph node dissection, lymph node metastasis, recurrent nerve chain lymph node, three-field dissection evidence of cervical lymph node metastasis. The remaining 31 patients had no recurrent nerve nodal metastasis on intraoperative pathological examination and therefore did not receive cervical lymph node dissection. None of these patients had cervical lymph node recurrence on follow-up. We compared patients who underwent intraoperative pathological investigation with those who underwent conventional 3 -field lymph node dissection (without performing intraoperative pathological investigation). The rates of cervical lymph node recurrence were similar among the groups $(2.6 \%$ vs. $6.7 \%$ ), but the 3-year survival rate was significantly higher in the patients who underwent intraoperative pathological dissection $(83.3 \%)$ than in those who underwent 3-field dissection $(57.2 \% ; \mathrm{p}<0.05)$. Although this was a retrospective study, our results suggest that outcomes of patients undergoing cervical lymph node dissection according to the results of intraoperative pathological investigation are at least as good as those in patients undergoing 3-field lymph node dissection. We conclude that intraoperative pathological investigation of recurrent nerve nodal metastasis is useful for determining whether cervical lymph node dissection should be performed in patients with thoracic esophageal cancer.

\section{Introduction}

Three-field lymph node dissection, comprising regional dissection of lymph nodes in the chest, abdomen, and neck, is one of the most commonly used procedures for the treatment of thoracic esophageal cancer. Good outcomes have consistently been obtained (1-5). However, this procedure has been criticized because it is very invasive and has a high incidence of complications such as recurrent nerve paralysis $(6,7)$. Whether 3 -field lymph node dissection should be performed in all patients with thoracic esophageal cancer remains controversial. Prospective identification of patients with thoracic esophageal cancer who are most likely to benefit from cervical lymph node dissection is an important clinical issue. 
Table I. Relationship between recurrent nerve nodal metastasis and cervical lymph node metastasis.

\begin{tabular}{lcccc}
\hline & & \multicolumn{2}{c}{ Cervical lymph node metastasis } & \\
\cline { 3 - 4 } Tumor location & No. of patients & Recurrent nerve nodal metastasis $(+)$ & Recurrent nerve nodal metastasis (-) & $\mathrm{p}$ value \\
\hline Ut & 32 & $3 / 11(27.3 \%)$ & $1 / 21(4.8 \%)$ & 0.1055 \\
Mt or Lt & 39 & $6 / 11(54.5 \%)$ & $4 / 28(14.3 \%)$ & $0.0167^{\mathrm{a}}$ \\
Total & 71 & $9 / 22(40.9 \%)$ & $5 / 49(10.2 \%)$ & $0.0074^{\mathrm{a}}$ \\
\hline
\end{tabular}

Statistical analysis: Fisher's exact tests. ${ }^{\mathrm{a}} \mathrm{p}<0.05$. Ut, upper thoracic esophagus; Mt, middle thoracic esophagus; Lt, lower thoracic esophagus.

Table II. Results of multiple logistic regression with the presence of cervical lymph node metastasis as the dependent variable.

\begin{tabular}{|c|c|c|c|c|c|}
\hline Independent variables & Estimate & Standard error & Odds ratio & $95 \% \mathrm{CI}$ & $\mathrm{p}$ value \\
\hline \multicolumn{6}{|l|}{ Gender } \\
\hline $\begin{array}{l}\text { Male } \\
\text { Female }\end{array}$ & 0.7349 & 0.699 & 2.085 & $0.530-8.207$ & 0.2931 \\
\hline Age (years) & 0.011 & 0.0489 & 1.011 & $0.919-1.113$ & 0.8213 \\
\hline $\begin{array}{l}\text { Location of tumor } \\
\text { Ut } \\
\text { Mt or Lt }\end{array}$ & 0.2639 & 0.5113 & 1.302 & $0.478-3.547$ & 0.6057 \\
\hline $\begin{array}{l}\text { Depth of tumor invasion } \\
\text { pTis-1 } \\
\text { pT2-4 }\end{array}$ & 0.4567 & 0.6638 & 1.579 & $0.430-5.799$ & 0.4914 \\
\hline No. of metastatic lymph nodes & 0.1686 & 0.1038 & 1.184 & $0.966-1.451$ & 0.1042 \\
\hline $\begin{array}{l}\text { Recurrent nerve nodal metastasi } \\
\text { Negative } \\
\text { Positive }\end{array}$ & 1.0905 & 0.49 & 2.976 & $1.139-7.775$ & $0.026^{\mathrm{a}}$ \\
\hline
\end{tabular}

Dependent variable: cervical lymph node metastasis. 95\% CI, 95\% confidence interval. ${ }^{\mathrm{a}} \mathrm{p}<0.05$. pT, pathological T-stage; Ut, upper thoracic esophagus; Mt, middle thoracic esophagus; Lt, lower thoracic esophagus.

Recently, sentinel-node navigation surgery has been introduced for the treatment of gastrointestinal cancer in an attempt to reduce the extent of lymph node dissection $(8,9)$. In esophageal cancer, recurrent nerve nodal metastasis is thought to be a predictor of cervical lymph node metastasis. Previous investigations have examined whether the decision to perform cervical lymph node dissection could be based on the presence or absence of recurrent nerve nodal metastasis on intraoperative pathological investigation (10-12). However, few studies have examined the relationship between treatment based on the results of intraoperative pathological investigation of recurrent nerve nodal metastasis and outcomes such as postoperative cervical lymph node recurrence and survival (13).

At our hospital, frozen sections of recurrent nerve lymph nodes have been pathologically examined during surgery since 2000 in patients with thoracic esophageal cancer. In patients without lymph node metastasis, cervical lymph node dissection is not performed. We retrospectively analyzed the relationship between recurrent nerve nodal metastasis and cervical lymph node metastasis to determine whether intraoperative patho- logical investigation was a useful procedure. We also compared the incidence of cervical lymph node recurrence and outcomes between patients undergoing intraoperative pathological investigation and those undergoing conventional 3-field lymph node dissection.

\section{Materials and methods}

Patients and surgical procedures. We retrospectively examined 102 patients with primary thoracic esophageal cancer who had undergone right transthoracic esophagectomy with extensive lymph node dissection (curative R0 resection) in the Department of Surgery, Division of Digestive Surgery, Kyoto Prefectural University of Medicine Graduate School of Medical Science, between January 1980 and December 2003. Of these patients, 61 underwent three-field lymph node dissection between 1980 and 2000. For cervical node dissection, the cervical lymph nodes, including the supraclavicular nodes and internal jugular nodes, were extensively dissected bilaterally (14). 
Table III. Clinicopathological features of 41 patients who underwent intraoperative pathological investigation of recurrent nerve nodal metastasis.

\begin{tabular}{lc}
\hline Variables & No. of cases \\
\hline Gender & 36 \\
Male & 5 \\
Female & $60.1 \pm 8.2$ \\
Age (years) & \\
Histological type & 39 \\
SCC & 2 \\
Other & \\
Location of tumor & 2 \\
Ut & 26 \\
Mt & 13 \\
Lt & \\
Depth of tumor invasion & 11 \\
pT1 & 30 \\
pT2-4 & $3.0 \pm 3.6$ \\
No. of metastatic lymph nodes & \\
pStage & 18 \\
0-II & 23 \\
III-IVa &
\end{tabular}

Mean \pm SD (standard deviation). SCC, squamous cell carcinoma; pStage, pathological stage; pT, pathological T-stage; Ut, upper thoracic esophagus; Mt, middle thoracic esophagus; Lt, lower thoracic esophagus.

Since 2000, we have performed cervical lymph node dissection according to the presence or absence of recurrent nerve nodal metastasis on intraoperative pathological examination in 41 patients with thoracic esophageal cancer. These patients underwent lymphadenectomy including the cervicothoracic, total mediastinal, perigastric, and celiac lymph nodes. Cervico-thoracic lymph node dissection around the recurrent laryngeal nerve was performed by a mediastinal approach (15). Lymph nodes were dissected bilaterally from the recurrent laryngeal nerves up to the inferior border of the thyroid gland. One lymph node located in the upper area was frozen rapidly and sliced into sections. The section with the maximum surface area was stained with hematoxylin and eosin (H\&E) and examined intraoperatively. Only patients who had recurrent nerve nodal metastasis underwent bilateral cervical lymph node dissection.

Clinicopathologic features. Various clinicopathologic features, including gender, age, number of metastatic lymph nodes, location of tumor, depth of tumor invasion, histological type, and pathological stage (pStage) were analyzed. Lymph nodes were fixed in formalin, embedded in paraffin, and stained with H\&E. One section of each lymph node was then examined histologically to determine the presence or absence of metastasis. Location of tumor, depth of tumor invasion, histological type, and pStage were classified according to the Japanese
Guide Lines for the Clinical and Pathologic Studies on Carcinoma of the Esophagus (16).

Statistical analysis. Statistical analysis was carried out using Student's t-tests and Fisher's exact tests for clinicopathological characteristics. Multiple logistic regression was used to identify risk factors associated with cervical lymph node metastases. Cumulative survival rates were calculated by the Kaplan-Meier method, and the statistical significance of differences was compared by the log-rank test. Differences were considered significant when the $p$ value was $<0.05$. These analyses were performed electronically with the use of statistical software (JMP, version 5)

\section{Results}

Relationship between recurrent nerve nodal metastasis and cervical lymph node metastasis. To investigate whether recurrent nerve nodal metastasis is a predictor of cervical lymph node metastasis in thoracic esophageal cancer, we analyzed the locations of metastatic lymph nodes as determined by $\mathrm{H} \& \mathrm{E}$ staining of paraffin sections obtained from 71 patients who underwent three-field lymph node dissection between 1980 and 2003. Ten of these patients underwent cervical lymph node dissection based on the results of intraoperative pathological investigations from 2000 onward. The numbers of patients with upper, middle, and lower thoracic esophageal cancer were 32,31 , and 8 , respectively.

Among 71 patients who underwent three-field lymph node dissection, cervical lymph node metastasis was found in 9 of 22 patients $(40.9 \%)$ with recurrent nerve nodal metastasis, as compared with only 5 of 49 patients $(10.2 \%)$ without recurrent nerve nodal metastasis. The presence of cervical lymph node metastasis strongly correlated with recurrent nerve nodal metastasis in patients who had middle or lower thoracic esophageal cancer, but not in those who had upper thoracic esophageal cancer (Table I).

Multiple logistic regression was performed with the presence of cervical lymph node metastasis as the dependent variable. Various clinicopathological features, such as gender, age, location of tumor, depth of tumor invasion, number of metastatic lymph nodes, and recurrent nerve nodal metastasis served as independent variables. Recurrent nerve nodal metastasis was found to be the strongest predictor of cervical lymph node metastasis (Table II)

These results suggested that the recurrent nerve lymph nodes are sentinel nodes that can be used to predict the likelihood of cervical lymph node metastasis in thoracic esophageal cancer.

Treatments and clinical courses of patients who underwent intraoperative pathological investigation of recurrent nerve nodal metastasis. Recurrent nerve nodal metastasis was pathologically examined during surgery in 41 patients between 2000 and 2003 (Table III). Cervical lymph node dissection was performed in the 10 patients in whom recurrent nerve nodal metastasis was diagnosed on intraoperative pathological examination. Two of these 10 patients were found to have cervical lymph node metastasis on histological examination of H\&E-stained paraffin sections. Of these 10 patients, cervical 


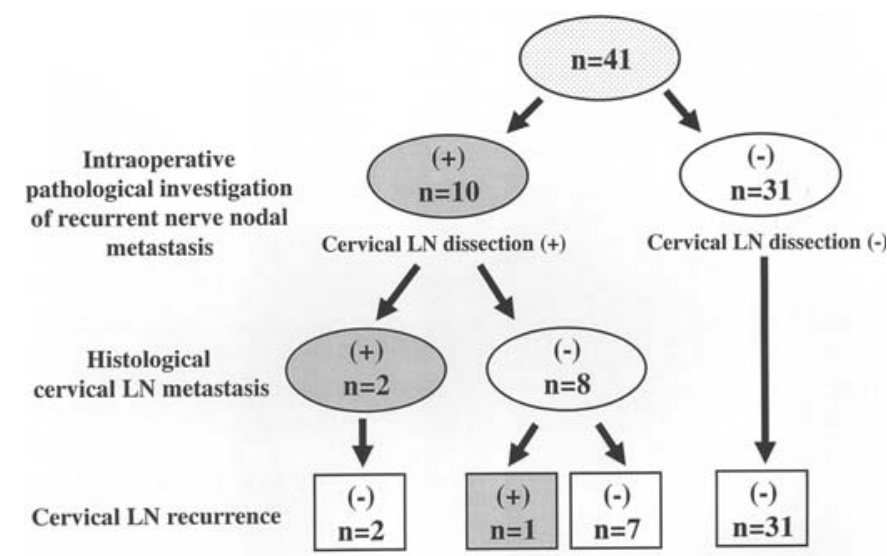

Figure 1. Flow chart showing the treatment procedure and clinical course of 41 patients who underwent intraoperative pathological investigation of recurrent nerve nodal metastasis. LN, lymph node.

lymph node recurrence occurred in only 1 who underwent cervical lymph node dissection because recurrent nerve nodal metastasis was positive on intraoperative pathological investigation, despite no evidence of cervical lymph node metastasis on histological examination of H\&E-stained paraffin sections. In contrast, no cervical lymph node recurrence was found in any of the 31 patients in whom cervical lymph node dissection was omitted because they were negative for recurrent nerve nodal metastasis on intraoperative pathological investigation (Fig. 1).

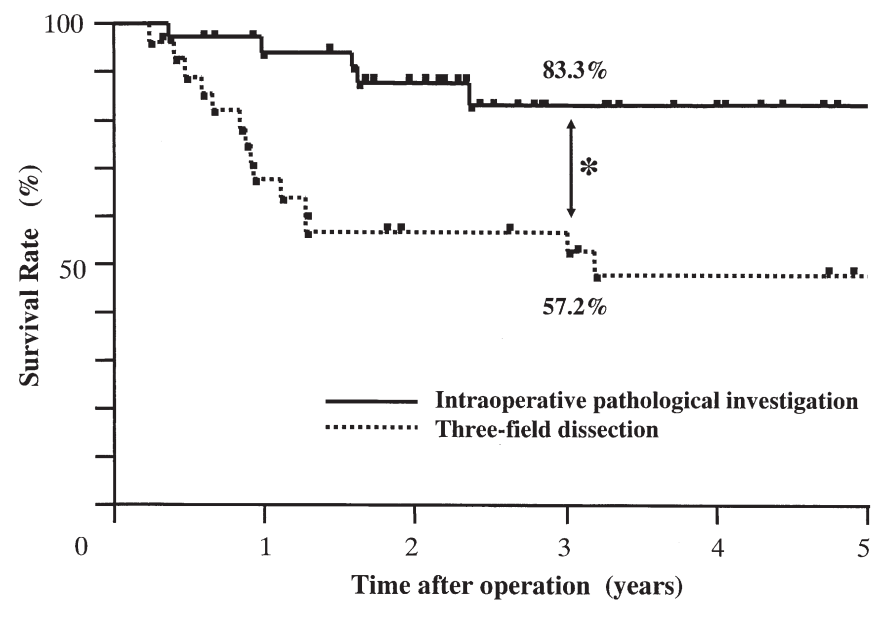

Figure 2. Survival rates of patients who underwent intraoperative pathological investigation of recurrent nerve nodal metastasis and of those who underwent conventional three-field lymph node dissection. Statistical analysis: log-rank test. ${ }^{*} \mathrm{p}<0.05$

Comparison of clinical outcomes between patients with cancer of the middle or lower thoracic esophagus who underwent lymph node dissection according to the results of intraoperative pathological investigation and those who underwent conventional three-field lymph node dissection. Finally, clinical outcomes of the 39 patients with cancer of the middle or lower thoracic esophagus who were treated according to the results of intraoperative pathological investigation of recurrent nerve nodal metastasis were compared with

Table IV. Comparison of clinicopathological features between patients with cancer of the middle or lower thoracic esophagus who underwent lymph node dissection according to the results of intraoperative pathological investigation and those who underwent conventional three-field lymph node dissection.

\begin{tabular}{|c|c|c|c|}
\hline Variables & Intraoperative investigation & Three-field dissection & $\mathrm{p}$ value \\
\hline \multicolumn{4}{|l|}{ Gender } \\
\hline Male & 35 & 22 & \multirow{2}{*}{0.1097} \\
\hline Female & 4 & 8 & \\
\hline Age (years) & $60.1 \pm 7.9$ & $58.3 \pm 7.9$ & 0.3434 \\
\hline \multicolumn{4}{|l|}{ Histological type } \\
\hline $\mathrm{SCC}$ & 37 & 29 & \multirow{2}{*}{1} \\
\hline Other & 2 & 1 & \\
\hline \multicolumn{4}{|l|}{ Location of tumor } \\
\hline Mt & 26 & 24 & \multirow{2}{*}{0.2815} \\
\hline $\mathrm{Lt}$ & 13 & 6 & \\
\hline \multicolumn{4}{|l|}{ Depth of tumor invasion } \\
\hline pT1 & 11 & 8 & \multirow[b]{2}{*}{1} \\
\hline pT2-4 & 28 & 22 & \\
\hline No. of metastatic lymph nodes & $2.9 \pm 3.7$ & $2.8 \pm 4.7$ & 0.9069 \\
\hline \multicolumn{4}{|l|}{ pStage } \\
\hline $0-\mathrm{II}$ & 18 & 14 & \multirow[b]{2}{*}{1} \\
\hline III-IVa & 21 & 16 & \\
\hline
\end{tabular}

Statistical analysis: Student's t-tests and Fisher's exact tests. Mean \pm SD. Mt, middle thoracic esophagus; Lt, lower thoracic esophagus. 
Table V. Comparison of cervical lymph node recurrence between patients with cancer of the middle or lower thoracic esophagus who underwent lymph node dissection according to the results of intraoperative pathological investigation and those who underwent conventional three-field lymph node dissection.

\begin{tabular}{lccc}
\hline & Intraoperative investigation & Three-field dissection & $\mathrm{p}$ value \\
\hline Cervical lymph node recurrence & $1 / 39(2.6 \%)$ & $2 / 30(6.7 \%)$ & 0.5757 \\
\hline
\end{tabular}

Statistical analysis: Fisher's exact tests.

those of the 30 patients who underwent conventional threefield lymph node dissection before 2000 , without undergoing intraoperative pathological investigation of recurrent nerve nodal metastasis. In this retrospective analysis, patients with upper thoracic esophageal cancer were excluded to decrease bias related to tumor location.

There were no significant differences in clinicopathological features between the two groups (Table IV). Cervical lymph node recurrence occurred in 1 of the 39 patients (2.6\%) who underwent intraoperative pathological investigation and in 2 of 30 patients $(6.7 \%)$ who underwent conventional three-field lymph node dissection. This difference was not significant $(\mathrm{p}=0.5757)$ (Table $\mathrm{V})$. The 3-year survival rates were $83.3 \%$ in patients who underwent intraoperative pathological investigation and $57.2 \%$ in those who underwent conventional three-field lymph node dissection $(\mathrm{p}<0.05)$ (Fig. 2),

These results suggest that outcomes in terms of both cervical lymph node recurrence and survival of patients with thoracic esophageal cancer, in whom cervical lymph node dissection was performed on the basis of whether recurrent nerve nodal metastasis was found on intraoperative pathological investigation, were at least as good as those of patients who underwent conventional three-field lymph node dissection.

\section{Discussion}

Recurrent nerve lymph nodes have the highest incidence of metastasis in patients with thoracic esophageal cancer. The status of these nodes is thus an important determinant of outcome $(17,18)$. Recurrent nerve lymph node dissection is thought to improve outcomes of patients with thoracic esophageal cancer. Anatomically, the recurrent nerve lymph nodes communicate with the cervical lymph nodes through lymphatic ducts (19-21). The recurrent nerve lymph nodes are therefore considered sentinel nodes of the cervical lymph nodes. Recent clinical studies have examined whether cervical lymph node dissection could be omitted in selected patients with thoracic esophageal cancer, instead of performing 3-field lymph node dissection in all patients. Tabira et al examined the relation between recurrent nerve nodal metastasis and cervical lymph node metastasis and reported that the rate of cervical lymph node metastasis was $43.5 \%$ in patients with recurrent nerve nodal metastasis, as compared with $11.1 \%$ in patients without such metastasis. Logistic-regression analysis demonstrated that recurrent nerve nodal metastasis is a useful predictor of cervical lymph node metastasis (10). Shiozaki et al reported that cervical lymph node metastasis was found in $51.6 \%$ of patients with recurrent nerve nodal metastasis, as compared with $11.6 \%$ of patients without recurrent nerve nodal metastasis (11). Our results are consistent with the findings of these previous studies and strongly suggest that recurrent nerve lymph nodes are sentinel nodes in patients with thoracic esophageal cancer.

Cervical lymph node metastasis was found on examination of paraffin sections in approximately $10 \%$ of patients without recurrent nerve nodal metastasis. This finding suggests that 'jumping metastasis' occurs in patients with thoracic esophageal cancer (22). Jumping metastasis is the main reason for recommending 3-field lymph node dissection for all patients with thoracic esophageal cancer. In our study, however, cervical lymph node recurrence did not occur in any of the 31 patients in whom cervical lymph node dissection was omitted because recurrent nerve nodal metastasis was negative on intraoperative pathological investigation. We previously showed that salvage cervical lymphadenectomy produced relatively good outcomes of patients with solitary supraclavicular lymph node recurrence (23). Judging from these findings, we recommend that the decision whether to perform cervical lymph node dissection is based on the results of intraoperative pathological investigation. Patients who subsequently have cervical lymph node recurrence should undergo salvage cervical lymphadenectomy. We believe that good outcomes can be obtained by following these steps.

To enhance the reliability of diagnosis, Noguchi et al postoperatively examined paraffin sections of recurrent nerve lymph nodes and performed cervical lymph node dissection by a two-step approach. They found that two-step three-field lymph node dissection was useful for the treatment of thoracic esophageal carcinoma (24). Dixon et al reported that the sensitivity of frozen-section analysis of axillary lymph nodes in patients with breast cancer was only $73 \%$ and therefore recommended against its routine use (25). In our study, however, the sensitivity of frozen-section analysis was $83.3 \%$ and the specificity was $100 \%$. The results of frozen-section analysis were thus not inferior to those of paraffin-section analysis (data not shown). Given the increased stress associated with two-step cervical lymph node dissection, we believe that one-step surgery with intraoperative pathological investigation offers considerable benefits.

Recently, techniques such as real-time polymerase-chainreaction (PCR) and immunohistochemistry have been used intraoperatively to detect micrometastasis to the recurrent nerve lymph nodes. Yoshioka et al reported that the results of intraoperative pathological investigation by frozen-section analysis were negative in 2 of 9 patients with cervical lymph 
astasis in whom the results of genetic diagnosis by node metastasis in whom the results of genetic diagnosis by
real-time PCR of recurrent nerve lymph node specimens were positive (13). Because the results of intraoperative pathological investigation were negative, these 2 patients did not undergo cervical lymph node dissection. Cervical lymph node recurrence subsequently developed in both of these patients. Nagatani et al reported that the detection rates of micrometastasis with hematoxylin and eosin staining, immunohistochemistry, and real-time PCR were $50.0 \%, 33.3 \%$, and $83.3 \%$, respectively. They recommended that intraoperative investigation should be performed with these 3 techniques (12). We agree that real-time PCR and immunohistochemical analysis are likely to increase the detection rate of recurrent nerve nodal metastasis, but these techniques can only be used at a limited number of hospitals (i.e., university and other large hospitals). In contrast, intraoperative pathological investigation is a more convenient technique that can be used at most hospitals where esophageal surgery is performed. Our results indicate that this technique provides excellent results in terms of detecting lymph node metastasis and leads to good outcomes in terms of cervical lymph node recurrence and survival. Our results suggest that intraoperative pathological investigation is currently the most effective method able to be routinely used clinically.

In conclusion, our results suggest that recurrent nerve nodal metastasis is a predictor of cervical lymph node metastasis in thoracic esophageal cancer. Our findings provide evidence that intraoperative pathological investigation of recurrent nerve nodal metastasis is a useful technique for deciding whether patients should undergo cervical lymph node dissection, resulting in outcomes at least as good as those with conventional 3-field lymph node dissection.

\section{References}

1. Kato H, Watanabe H, Tachimori Y and Iizuka T: Evaluation of neck lymph node dissection for thoracic esophageal carcinoma. Ann Thorac Surg 51: 931-935, 1991.

2. Akiyama H, Tsurumaru M, Udagawa $H$ and Kajiyama Y: Radical lymph node dissection for cancer of the thoracic esophagus. Ann Surg 220: 364-373, 1994.

3. Baba M, Aikou T, Yoshinaka H, Natsugoe S, Fukumoto T, Shimazu $\mathrm{H}$ and Akazawa K: Long-term results of subtotal esophagectomy with three-field lymphadenectomy for carcinoma of the thoracic esophagus. Ann Surg 219: 310-316, 1994.

4. Fujita H, Kakegawa T, Yamana H, Shima I, Toh Y, Tomita Y, Fujii T, Yamasaki K, Higaki K, Noake T, Ishibashi N and Mizutani K: Mortality and morbidity rates, postoperative course, quality of life, and prognosis after extended radical lymphadenectomy for esophageal cancer. Comparison of threefield lymphadenectomy with two-field lymphadenectomy. Ann Surg 222: 654-662, 1995 .

5. Altorki N, Kent M, Ferrara C and Port J: Three-field lymph node dissection for squamous cell and adenocarcinoma of the esophagus. Ann Surg 236: 177-183, 2002.

6. Isono K, Sato H and Nakayama K: Results of a nationwide study on the three-field lymph node dissection of esophageal cancer. Oncology 48: 411-420, 1991.

7. Nishimaki T, Suzuki T, Suzuki S, Kuwabara S and Hatakeyama K: Outcomes of extended radical esophagectomy for thoracic esophageal cancer. J Am Coll Surg 186: 306-312, 1998.
8. Kitagawa Y and Kitajima M: Gastrointestinal cancer and sentinel node navigation surgery. J Surg Oncol 79: 188-193, 2002.

9. Kitagawa Y, Fujii H, Mukai M, Kubota T, Ando N, Watanabe M Ohgami M, Otani Y, Ozawa S, Hasegawa H, Furukawa T, Kumai K, Ikeda T, Nakahara T, Kubo A and Kitajima M: The role of the sentinel lymph node in gastrointestinal cancer. Surg Clin North Am 80: 1799-1809, 2000.

10. Tabira Y, Yasunaga M, Tanaka M, Nakano K, Sakaguchi T, Nagamoto N, Ogi S and Kitamura N: Recurrent nerve nodal involvement is associated with cervical nodal metastasis in thoracic esophageal carcinoma. J Am Coll Surg 191: 232-237, 2000 .

11. Shiozaki H, Yano M, Tsujinaka T, Inoue M, Tamura S, Doki Y Yasuda T, Fujiwara Y and Monden M: Lymph node metastasis along the recurrent nerve chain is an indication for cervical lymph node dissection in thoracic esophageal cancer. Dis Esophagus 14: 191-196, 2001.

12. Nagatani S, Shimada Y, Li Z, Kaganoi J, Kan T, Maeda M, Watanabe $\mathrm{G}$ and Imamura $\mathrm{M}$ : Validation of intra-operative detection of paratracheal lymph node metastasis using real-time RT-PCR targeting esophageal squamous cell carcinoma. Jpn J Clin Oncol 33: 549-555, 2003.

13. Yoshioka S, Fujiwara Y, Sugita Y, Okada Y, Yano M, Tamura S, Yasuda T, Takiguchi S, Shiozaki H and Monden M: Real-time rapid reverse transcriptase-polymerase chain reaction for intraoperative diagnosis of lymph node micrometastasis: clinical application for cervical lymph node dissection in esophageal cancers. Surgery 132: 34-40, 2002.

14. Akiyama H: Surgery for Cancer of the Esophagus. Williams \& Wilkins, Baltimore, pp19-42, 1990.

15. Watanabe H: The problems of cervico-thoraco abdominal (3field) lymphadenectomy for thoracic esophageal cancer. Ann Thorac Cardiovasc Surg 1: 349-353, 1995.

16. Japanese Society for Esophageal Disease: Guide Lines for the Clinical and Pathologic Studies on Carcinoma of the Esophagus. 9th edition. 1999.

17. Fujita H, Kakegawa T, Yamana H, Shima I, Tanaka H, Ikeda S, Nogami S and Toh Y: Lymph node metastasis and recurrence in patients with a carcinoma of the thoracic esophagus who underwent three-field dissection. World J Surg 18: 266-272, 1994.

18. Malassagne B, Tiret E, Duprez D, Coste J, de Sigalony JP and Parc R: Prognostic value of thoracic recurrent nerve nodal involvement in esophageal squamous cell carcinoma. J Am Coll Surg 185: 244-249, 1997.

19. Sato T and Iizuka T: Color Atlas of Surgical Anatomy for Esophageal Cancer. Springer-Verlag, Tokyo, pp25-90, 1992

20. Tanabe G, Baba M, Kuroshima K, Natugoe S, Yoshinaka H, Aikou T and Kajisa T: Clinical evaluation of the esophageal lymph flow system based on RI uptake of dissected regional lymph nodes following lymphoscintigraphy. J Jpn Surg Soc 87: 315-323, 1986 .

21. Kutsuna M: Intrathoracic lymph flow, with special regard to the communication of intrathoracic lymph vessels with cervical lymph nodes. Kumamoto Med J 18: 228-233, 1965.

22. Sannohe Y, Hiratsuka R and Doki K: Lymph node metastases in cancer of the thoracic esophagus. Am J Surg 141: 216-218, 1981.

23. Komatsu S, Shioaki Y, Ichikawa D, Hamashima T, Kan K, Ueshima Y, Koide K, Lee CJ, Ikeda E, Mutoh F, Kurioka H, Oka T and Yamagishi H: Survival and clinical evaluation of salvage operation for cervical lymph node recurrence in esophageal cancer. Hepatogastroenterology 52: 796-799, 2005.

24. Noguchi T, Wada S, Takeno S, Hashimoto T, Moriyama H and Uchida Y: Two-step three-field lymph node dissection is beneficial for thoracic esophageal carcinoma. Dis Esophagus 17: 27-31, 2004.

25. Dixon JM, Mamman U and Thomas J: Accuracy of intraoperative frozen-section analysis of axillary nodes. Edinburgh Breast Unit team. Br J Surg 86: 392-395, 1999. 